\title{
Design of Two-Dimensional Digital Filters by Using the Singular Value Decomposition
}

\author{
ANDREAS ANTONIOU, FELLOW, IEEE, AND WU-SHENG LU, MEMBER, IEEE
}

\begin{abstract}
A method for the design of quadrantally symmetric 2-D digital filters is described. Like the method outlined by Twogood and Mitra, the method proposed here is based on the singular value decomposition. The salient difference between the two methods is that the proposed method uses one or more parallel correction sections which reduce the approximation error. In this way, reduced passband ripple and increased minimum stopband attenuation can easily he achieved. An important merit of the method is that the required 2-D filter is decomposed into a set of 1-D digital subfilters, which are much easier to design by optimization than the original 2-D filter.
\end{abstract}

\section{INTRODUCTION}

$\mathrm{T}$ HE DESIGN OF two-dimensional (2-D) digital filters has been a subject of study since the early 1970's. It is now widely known that the design can be carried out by applying transformations to $1-\mathrm{D}$ analog or digital transfer functions [1]-[6], by using various optimization methods [7]-[9], by applying transformations in conjunction with optimization methods [10], [11], or by using the singular value decomposition (SVD) [12], [13]. In [12], the SVD is applied to the so-called planar response matrix, which is obtaincd by truncating the impulse response array. The approach yields a class of multistage separable planar filters. In [13], the SVD is used to express a specified 2-D sampled amplitude response into a sum of products. The outcome in this case is a class of 2-D digital filters in which the mean-square error is minimized. While the method yields stable and computationally efficient filters for some highly nonseparable characteristics (e.g., circularly symmetric and fan characteristics), the resulting approximation error is large both in the passband and in the stopband.

In this paper, an improved method for the design of quadrantally symmetric 2-D digital filters is proposed. Like the method of Twogood and Mitra [13], the method proposed uses the SVD in conjunction with various wellknown techniques for the design of 1-D digital filters. The method leads to a set of stable and separable subfilters which can be connected in parallel to yield the desired 2-D digital filter. As will be demonstrated by designing a circularly symmetric 2-D filter, the method leads to reduced passband ripple and increased minimum stopband attenuation. Further, the degree of circularity achieved is

Manuscript received July 28, 1986; revised January 15, 1987. This work was supported in part by the Natural Sciences and Engineering Research Council of Canada under Grant A5802.

The authors are with the Department of Electrical and Computer Engineering, University of Victoria, Victoria, B.C., Canada V8W 2 Y2.

IEEE Log Number 8715934.

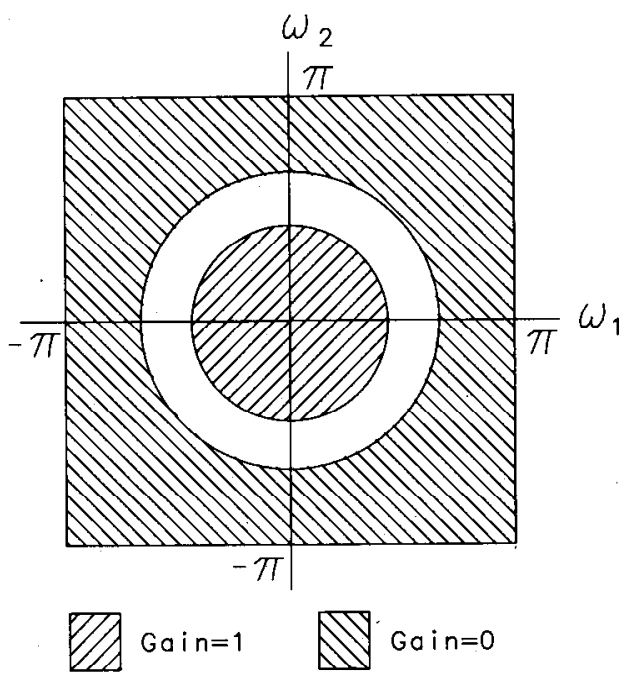

Fig. 1. Idealized amplitude response of quadrantally symmetric low-pass digital filter.

good, and the designs obtained are quite economical in terms of the amount of computation required for the implementation.

\section{Method of Twogood And Mitra}

A 2-D digital filter characterized by a transfer function $H\left(z_{1}, z_{2}\right)$ is said to be quadrantally symmetric if

$$
\begin{aligned}
\left|H\left(e^{j \omega_{1} T_{1}}, e^{j \omega_{2} T_{2}}\right)\right| & =\left|H\left(e^{-j \omega_{1} T_{1}}, e^{j \omega_{2} T_{2}}\right)\right| \\
& =\left|H\left(e^{j \omega_{1} T_{1}}, e^{-j \omega_{2} T_{2}}\right)\right| \\
& =\left|H\left(e^{-j \omega_{1} T_{1}}, e^{-j \omega_{2} T_{2}}\right)\right| .
\end{aligned}
$$

Two examples of quadrantally symmetric filters are the circularly symmetric filter and the fan filter. The idealized amplitude response of a circularly symmetric low-pass filter is depicted in Fig. 1.

In a quadrantally symmetric filter, $H\left(z_{1}, z_{2}\right)$ has a separable denominator [14]; therefore, it can be expressed as

$$
H\left(z_{1}, z_{2}\right)=\sum_{i=1}^{k} f_{i}\left(z_{1}\right) g_{i}\left(z_{2}\right)
$$

In effect, a quadrantally symmetric filter can always be realized using a set of $k$ parallel sections where the $i$ th section is a separable subfilter characterized by the transfer function $f_{i}\left(z_{1}\right) g_{i}\left(z_{2}\right)$. 
Let $A=\left(a_{l m}\right)$ be a desired amplitude response where

$$
a_{l m}=\left|H\left(e^{j \pi \mu_{l}}, e^{j \pi v_{m}}\right)\right|, \quad 1 \leqslant l \leqslant L, \quad 1 \leqslant m \leqslant M
$$

and let $\mu_{l}$ and $\nu_{m}$ be normalized frequencies such that

$$
\mu_{l}=\frac{l-1}{L-1} \quad \text { and } \quad \nu_{m}=\frac{m-1}{M-1}
$$

and $0 \leqslant \mu_{l} \leqslant 1,0 \leqslant \nu_{m} \leqslant 1$. The SVD of $A$ gives

$$
A=\sum_{i=1}^{r} \sigma_{i} u_{i} v_{i}^{\prime}
$$

where $\sigma_{1} \geqslant \sigma_{2} \geqslant \cdots \geqslant \sigma_{r}$ are the singular values of $A, u_{i}$ is the $i$ th eigenvector of $A A^{\prime}$ associated with the $i$ th eigenvalue $\sigma_{i}^{2}, v_{i}$ is the $i$ th eigenvector of $A^{\prime} A$ associated with $\sigma_{i}^{2}, r$ is the rank of $A$, and $v_{i}^{\prime}$ denotes the transpose of $v_{i}[15]$. If we let $\phi_{i}=\sigma_{i}^{1 / 2} u_{i}$ and $\gamma_{i}=\sigma_{i}^{1 / 2} v_{i}$, then (4) can be written as

$$
A=\sum_{i=1}^{r} \phi_{i} \gamma_{i}^{\prime}
$$

where $\left\{\phi_{i}, 1 \leqslant i \leqslant r\right\}$ and $\left\{\gamma_{i}, 1 \leqslant i \leqslant r\right\}$ are sets of orthogonal $L$-dimensional and $M$-dimensional vectors, respectively.

An important property of the SVD can be stated as

$$
\left\|A-\sum_{i=1}^{k} \phi_{i} \gamma_{i}^{\prime}\right\|=\min _{\hat{\phi}_{i}, \hat{\gamma}_{i}}\left\|A-\sum_{i=1}^{k} \hat{\phi}_{i} \hat{\gamma}_{i}^{\prime}\right\| \quad \text { for } 1 \leqslant k \leqslant r
$$

where $\hat{\phi}_{i} \in R^{L}, \hat{\gamma}_{i} \in R^{M}$, and

$$
\|X\|=\left[\sum_{l=1}^{L} \sum_{m=1}^{M} x_{l m}^{2}\right]^{1 / 2}
$$

is the Frobenius norm of a matrix $X=\left(x_{l m}\right) \in R^{L \times M}$. The above relation shows that for any fixed $k(1 \leqslant k$ $\leqslant r), \sum_{i=1}^{k} \phi_{i} \gamma_{i}^{\prime}$ is a minimal mean-square-error approximation to $A$.

Since all entries of $A$ are nonnegative, it follows that all entrics of $\phi_{1}$ and $\gamma_{1}$ are nonnegative [13], [16]. Nevertheless, the elements of $\phi_{i}$ and $\gamma_{i}$ for $i \geqslant 2$ may assume negative values.

Equation (5) can be written as

$$
A=\phi_{1} \gamma_{1}^{\prime}+\epsilon_{1}
$$

where

$$
\epsilon_{1}=\sum_{i=2}^{r} \phi_{i} \gamma_{i}^{\prime}
$$

Now on comparing (7) with (2) and assuming that $k=1$ and that $\phi_{1}$ and $\gamma_{1}$ are the desired amplitude responses for the 1-D filters characterized by $f_{1}\left(z_{1}\right)$ and $g_{1}\left(z_{2}\right)$, respectively, a 2-D digital filter can be designed through the following steps.

(1) Design 1-D filters $f_{1}$ and $g_{1}$ characterized by $f_{1}\left(z_{1}\right)$ and $g_{1}\left(z_{2}\right)$.

(2) Connect filters $f_{1}$ and $g_{1}$ in cascade.

Step (1) can be carried out by using one of the many available optimization methods. When filters $f_{1}$ and $g_{1}$ are designed, we have

$$
\left|f_{1}\left(e^{j \pi \mu l}\right)\right| \approx \phi_{1 l}, \quad 1 \leqslant l \leqslant L
$$

and

$$
\left|g_{1}\left(e^{j \pi \nu_{m}}\right)\right| \approx \gamma_{1 m}, \quad 1 \leqslant m \leqslant M
$$

where $\phi_{1 l}$ and $\gamma_{1 m}$ denote the $l$ th component of $\phi_{1}$ and the $m$ th component of $\gamma_{1}$, respectively. The transfer function of the cascade filter obtained in step (2) is given by

$$
H_{1}\left(z_{1}, z_{2}\right)=f_{1}\left(z_{1}\right) g_{1}\left(z_{2}\right)
$$

where

$$
\left(\left|H_{1}\left(e^{j \pi \mu_{l}}, e^{j \pi \nu_{m}}\right)\right|\right) \approx\left(\phi_{1 /} \gamma_{1 m}\right)=\phi_{1} \gamma_{1}^{\prime}
$$

and from (6)

$$
\left\|A-\left|H_{1}\left(e^{j \pi \pi \mu_{l}}, e^{j \pi \nu_{m}}\right)\right|\right\| \approx\left\|A-\phi_{1} \gamma_{1}^{\prime}\right\|=\epsilon_{1} .
$$

In effect, the amplitude response of the filter obtained is a minimal mean-square-error approximation to the desired amplitude response. The main disadvantage of the method is that error $\epsilon_{1}$ is usually relatively large and the filter might not be acceptable.

It should be mentioned here that there are several other matrix decompositions which will convert $A$ into a sum of products as in (5), e.g. the Jordan and LU decompositions [17]. However, only the SVD has the highly desirable property stated in (6).

\section{IMPROVED METHOD}

The method of Twogood and Mitra can be improved by finding a way of realizing more of the terms in (5) by means of parallel filter sections so that the approximation error may be reduced. From (5), we can write

$$
A=\phi_{1} \gamma_{1}^{\prime}+\phi_{2} \gamma_{2}^{\prime}+\epsilon_{2}
$$

where

$$
\epsilon_{2}=\sum_{i=3}^{r} \phi_{i} \gamma_{i}^{\prime} .
$$

Since $\phi_{2}$ and $\gamma_{2}$ may have negative components, a careful treatment of the second term in (8) is necessary.

Let $\phi_{2}^{-}$and $\gamma_{2}^{-}$be the absolute values of the most negative components of $\phi_{2}$ and $\gamma_{2}$, respectively. If

$$
e_{\phi}=\left[\begin{array}{ll}
1 & 1 \cdots 1
\end{array}\right]^{\prime} \in R^{L} \text { and } e_{\gamma}=\left[\begin{array}{lll}
1 & 1 & \cdots
\end{array}\right]^{\prime} \in R^{M}
$$

then all components of

$$
\tilde{\phi}_{2}=\phi_{2}+\phi_{2}^{-} e_{\phi} \text { and } \tilde{\gamma}_{2}=\gamma_{2}+\gamma_{2}^{-} e_{\gamma}
$$

are nonnegative. Let us assume that it is possible to design 1-D linear-phase or zero-phase filters characterized by $\tilde{f_{1}}\left(z_{1}\right), \tilde{g}_{1}\left(z_{2}\right), \tilde{f_{2}}\left(z_{1}\right)$, and $\tilde{g}_{2}\left(z_{2}\right)$ such that

$$
\begin{aligned}
& \tilde{f_{i}}\left(e^{j \pi \mu_{l}}\right)=\left|\tilde{f_{i}}\left(e^{j \pi \mu_{l}}\right)\right| e^{j \alpha_{1} \mu_{l}}, \quad 1 \leqslant l \leqslant L, \quad i=1,2 \\
& \tilde{g}_{i}\left(e^{j \pi \nu_{m}}\right)=\left|\tilde{g}_{i}\left(e^{j \pi \nu_{m}}\right)\right| e^{j \alpha_{2} \nu_{m}}, \quad 1 \leqslant m \leqslant M, \quad i=1,2
\end{aligned}
$$


where

$$
\begin{gathered}
\left|\tilde{f}_{1}\left(e^{j \pi \mu_{l}}\right)\right| \approx \phi_{1 l} \\
\left|\tilde{f}_{2}\left(e^{j \pi \mu_{l}}\right)\right| \approx \tilde{\phi}_{2 l} \\
\left|\tilde{g}_{1}\left(e^{j \pi \nu_{m}}\right)\right| \approx \gamma_{1 m} \\
\left|\tilde{g}_{2}\left(e^{j \pi \nu_{m}}\right)\right| \approx \tilde{\gamma}_{2 m} .
\end{gathered}
$$

Here, $\tilde{\phi}_{2 l}$ and $\tilde{\gamma}_{2 m}$ are the $l$ th component of $\tilde{\phi}_{2}$ and the $m$ th component of $\tilde{\gamma}_{2}$, respectively, and $\alpha_{1}, \alpha_{2}$ in (10) are constants which are equal to zero if zero-phase filters are to be employed. Now let

$$
\alpha_{1}=-\pi n_{1} \text { and } \alpha_{2}=-\pi n_{2}
$$

where $n_{1}, n_{2}$ are nonnegative integers, and define

$$
f_{2}\left(z_{1}\right)=\tilde{f_{2}}\left(z_{1}\right)-\phi_{2}^{-} z_{1}^{-n_{1}}
$$

and

$$
g_{2}\left(z_{2}\right)=\tilde{g}_{2}\left(z_{2}\right)-\gamma_{2} z_{2}^{-n_{2}} \text {. }
$$

From (9)-(13), it follows that

$$
f_{2}\left(e^{j \pi \mu_{l}}\right)=\left(\left|\tilde{f_{2}}\left(e^{j \pi \mu_{l}}\right)\right|-\phi_{2}^{-}\right) e^{-j \pi n_{1} \mu_{l}} \approx \phi_{2 l} e^{-j \pi n_{1} \mu_{l}},
$$

and

$$
\begin{array}{r}
g_{2}\left(e^{j \pi v_{m}}\right)=\left(\left|\tilde{g}_{2}\left(e^{j \pi v_{m}}\right)\right|-\gamma_{2}^{-}\right) e^{-j \pi n_{2} \nu_{m}} \approx \gamma_{2 m} e^{-j \pi n_{2} v_{m}}, \\
1 \leqslant m \leqslant M .
\end{array}
$$

Moreover, if we form

$$
H_{2}\left(z_{1}, z_{2}\right)=f_{1}\left(z_{1}\right) g_{1}\left(z_{2}\right)+f_{2}\left(z_{1}\right) g_{2}\left(z_{2}\right)
$$

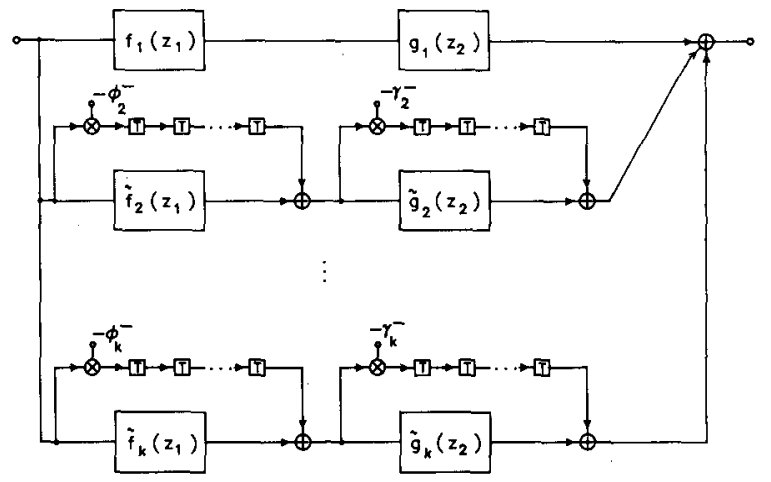

Fig. 2. Realization of quadrantally symmetric $2-D$ filter.

represents the main contribution to the amplitude response of the 2-D filter. For this reason, the subfilter characterized by $f_{1}\left(z_{1}\right) g_{1}\left(z_{2}\right)$ is said to be the main section of the 2-D filter. On the other hand,

$$
\left|f_{2}\left(e^{j \pi \mu_{i}}\right) g_{2}\left(e^{j \pi \nu_{m}}\right)\right|
$$

represents a correction to the amplitude response, and the subfilter characterized by $f_{2}\left(z_{1}\right) g_{2}\left(z_{2}\right)$ is said to represent a correction section.

Through the use of data $\phi_{i}$ and $\gamma_{i}(i=3, \cdots, k, k \leqslant r)$ given in (5), vectors $\tilde{\phi}_{i}$ and $\tilde{\gamma}_{i}$ can be found, and correction sections characterized by $f_{i}\left(z_{1}\right) g_{i}\left(z_{2}\right)$ can then be designed in a similar manner. When $k$ sections are designed, including the main section, $H_{k}\left(z_{1}, z_{2}\right)$ can be formed as

$$
H_{k}\left(z_{1}, z_{2}\right)=\sum_{i=1}^{k} f_{i}\left(z_{1}\right) g_{i}\left(z_{2}\right)
$$

and from (6), we have

$$
\left\|A-\left|H_{k}\left(e^{j \pi \mu_{1}}, e^{j \pi \nu_{m}}\right)\right|\right\| \approx\left\|A-\left|\sum_{i=1}^{k} \phi_{i} \gamma_{i}^{\prime}\right|\right\| \leqslant \epsilon_{k}=\min _{\hat{\phi}_{i}, \hat{\gamma}_{i}}\left\|A-\sum_{i=1}^{k} \hat{\phi}_{i} \hat{\gamma}_{i}^{\prime}\right\| .
$$

then

$$
\begin{aligned}
& \left|H_{2}\left(e^{j \pi \mu_{l}}, e^{j \pi \nu_{m}}\right)\right| \\
& \quad=\left|f_{1}\left(e^{j \pi \mu_{l}}\right) g_{1}\left(e^{j \pi \nu_{m}}\right)+f_{2}\left(e^{j \pi \mu_{l}}\right) g_{2}\left(e^{j \pi \nu_{m}}\right)\right| \\
& \quad \approx\left|\phi_{1 l} \gamma_{1 m}+\phi_{2 l} \gamma_{2 m}\right|, \quad 1 \leqslant l \leqslant L, \quad 1 \leqslant m \leqslant M
\end{aligned}
$$

In effect, a 2-D digital filter comprising $k$ sections is obtained whose amplitude response is a minimal meansquare-error approximation to the desired amplitude response.

which in conjunction with (6) implies that

$$
\begin{aligned}
\left\|A-\left|H_{2}\left(e^{j \pi \mu_{l}}, e^{j \pi v_{m}}\right)\right|\right\| & \approx\left\|A-\left|\phi_{1} \gamma_{1}^{\prime}+\phi_{2} \gamma_{2}^{\prime}\right|\right\| \\
& \leqslant\left\|A-\left(\phi_{1} \gamma_{1}^{\prime}+\phi_{2} \gamma_{2}^{\prime}\right)\right\|=\epsilon_{2}=\min _{\hat{\phi}_{i}, \hat{\gamma}_{i}}\left\|A-\left(\hat{\phi}_{1} \hat{\gamma}_{1}^{\prime}+\hat{\phi}_{2} \hat{\gamma}_{2}^{\prime}\right)\right\| .
\end{aligned}
$$

Evidently, through the above technique it is possible to realize the second term in (5) by means of a parallel subfilter, thereby reducing the approximation error from $\epsilon_{1}$ to $\epsilon_{2}$. According to (15), the two-section 2-D digital filter obtained has an amplitude response which is a minimal mean-square-error approximation to the desired amplitude response.

Since $f_{1}\left(z_{1}\right) g_{1}\left(z_{2}\right)$ corresponds to the largest singular value $\sigma_{1}$, the quantity

$$
\left|f_{1}\left(e^{j \pi \mu_{t}}\right) g_{1}\left(e^{j \pi \nu_{m}}\right)\right|
$$

The method leads to an asymptotically stable 2-D filter, provided that the various 1-D subfilters are stable. This requirement is easily satisfied in practice.

The general structure of the 2-D filter obtained is illustrated in Fig. 2, where the various 1-D subfilters may be either linear-phase or zero-phase filters, as was shown earlier. Evidently, the structure obtained is a parallel arrangement of cascade low-order sections; consequently, the traditional advantages associated with parallel and/or cascade structures apply. First, the sensitivity of the struc- 


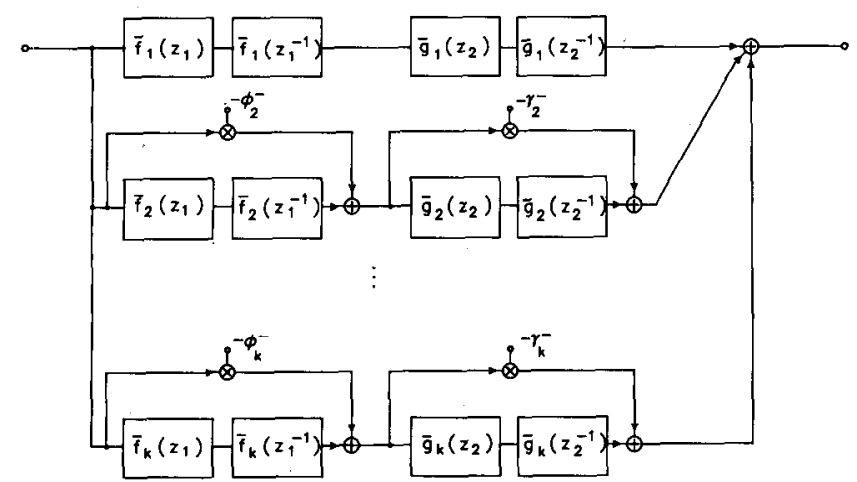

Fig. 3. Realization of quadrantally symmetric $2-D$ filter using zero-phase subfilters.

ture to coefficient quantization is expected to be low. Second, computations in the various parallel subfilters can be carried out simultaneously. Third, the number of multipliers (or multiplications per output sample) is small. For example, if there were $k$ parallel subfilters in Fig. 2 and each was of order $N \times N$, then the upper bound on the number of multipliers would be $4 k(N+1)$ as opposed to $(k N+1)^{2}+2 k N$ in the case of a corresponding state-space implementation.

If linear-phase subfilters are to be employed, the equalities in (12) must be satisfied. This implies that the subfilters must have constant group delays which are multiples of $T_{1}$ or $T_{2}$. Causal subfilters of this class can be designed as nonrecursive filters by using the weightedChebyshev approximation method [18], [19]. In such a case, the 2-D filter obtained can be used in real-time applications.

If a record of the data to be processed is available, the processing can be carried out in nonreal time. In such a case, the subfilters in Fig. 2 can be designed as zero-phase recursive filters. The resulting structure is depicted in Fig. 3 , where $\bar{f}_{i}\left(z_{1}\right)$ and $\bar{f}_{i}\left(z_{1}^{-1}\right)\left(\bar{g}_{i}\left(z_{2}\right)\right.$ and $\left.\bar{g}_{i}\left(z_{2}^{-1}\right)\right)$ contribute equally to the amplitude response of the 2-D filter. The design can be completed by assuming that the desired amplitude responses for subfilters $\bar{f}_{1}, \bar{g}_{1}, \bar{f}_{i}$, and $\bar{g}_{i}$ for $i=2, \cdots, k$ are $\phi_{1}^{1 / 2}, \gamma_{1}^{1 / 2}, \phi_{i}^{1 / 2}$, and $\gamma_{i}^{1 / 2}$ for $i=2, \cdots, k$, respectively. The various subfilters can readily be designed by using the minimax optimization method described in [20] and [21]. This method may yield unstable 1-D digital filters; but the problem can readily be eliminated by replacing poles outside the unit circle by their reciprocals and then adjusting the multiplier constant of the transfer function [22]. The advantage of using zero-phase recursive subfilters is that 2-D filters of high selectivity and low computational complexity can readily be designed.

It should be mentioned here that it would be possible in principle to use linear-phase recursive subfilters in the design. This approach would lead to high selectivity 2-D filters with low computational complexity, which could be used in real-time applications. Unfortunately, however, linear-phase recursive filters with group delays which are multiples of $T_{1}$ or $T_{2}$ are difficult to design in practice.

When a circularly symmetric 2-D filter is required, the design work can be reduced significantly. Matrix $A$ defined

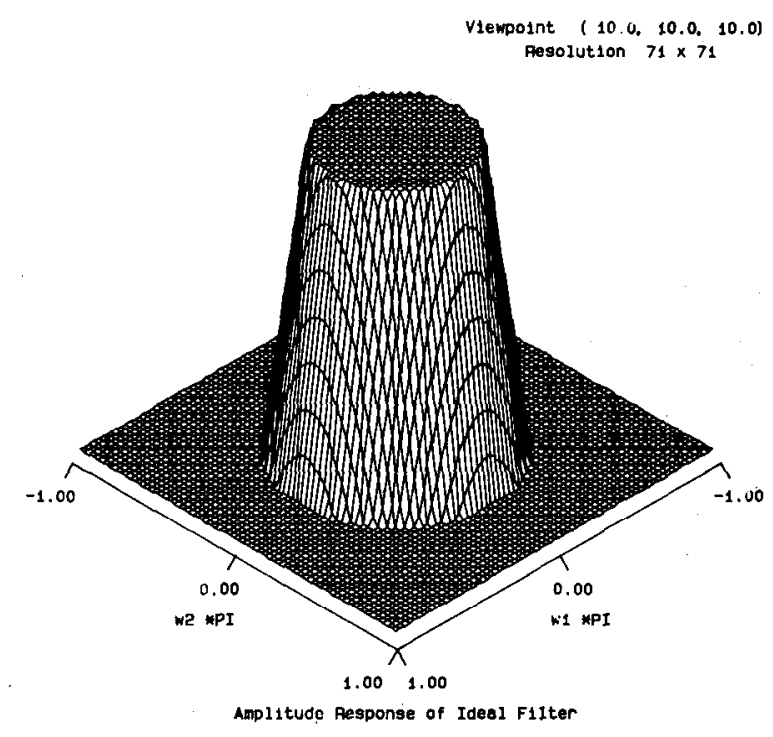

Fig. 4. Ideal amplitude response of low-pass filter.

in (3) is symmetric; therefore, (5) becomes

$$
A=\sum_{i=1}^{r} s_{i} \phi_{i} \phi_{i}^{\prime}
$$

where $s_{1}=1$ and $s_{i}= \pm 1$ for $2 \leqslant i \leqslant r$. This shows that each parallel section requires only one 1-D subfilter to be designed and as a consequence the design work is reduced by 50 percent. The realization remains as in Fig. 2 or 3 except that

$$
g_{i}\left(z_{2}\right)=\left.f_{i}\left(z_{1}\right)\right|_{z_{1}=z_{2}}
$$

\section{ERror COMPENSATION}

The method described in the preceding section leads to good results. Nevertheless, a further improvement is possible through the use of error compensation.

When the main section and the correction sections are designed by using an optimization method, approximation errors will inevitably occur which will accumulate and manifest themselves as the overall approximation error in the design of the 2-D filter. Fortunately, it is possible to prevent the accumulation of error through compensation.

When the design of the main section is complete, define an error matrix

$$
E_{1}=A-\left|f_{1}\left(e^{j \pi \mu_{l}}\right) g_{1}\left(e^{j \pi \nu_{m}}\right)\right|
$$

and then perform SVD on $E_{1}$ to obtain

$$
E_{1}=s_{22} \dot{\phi}_{22} \gamma_{22}^{\prime}+\cdots+s_{r 2} \phi_{r 2} \gamma_{r 2}^{\prime} \text {. }
$$

Data $\phi_{22}$ and $\gamma_{22}^{\prime}$ in (20) can be used to deduce $f_{2}\left(z_{1}\right) g_{2}\left(z_{2}\right)$ as in Section III; thus, the first correction section can be designed. Now form error matrix $E_{2}$ as

$$
\begin{aligned}
& E_{2}=E_{1}-\left|s_{22} f_{2}\left(e^{j \pi \mu_{f}}\right) g_{2}\left(e^{j \pi v_{m}}\right)\right| \\
& =A-\left|f_{1}\left(e^{j \pi \mu_{l}}\right) g_{1}\left(e^{j \pi v_{m}}\right)+s_{22} f_{2}\left(e^{j \pi \mu_{i}}\right) g_{2}\left(e^{j \pi \nu_{m}}\right)\right|
\end{aligned}
$$

and perform SVD on $E_{2}$ to obtain

$$
E_{2}=s_{33} \phi_{33} \gamma_{33}^{\prime}+\cdots+s_{r 3} \phi_{r 3} \gamma_{r 3}^{\prime} \text {. }
$$


As before, data $\phi_{33}$ and $\gamma_{33}^{\prime}$ can be used to design the second correction section. This procedure is continued until the elements of the error matrix become sufficiently small for the application at hand.

\section{A Design Example}

In this section, we illustrate the proposed method by designing a circularly symmetric, zero-phase, 2-D filter specified by

$$
\left|H\left(e^{j \omega_{1} T_{1}}, e^{j \omega_{2} T_{2}}\right)\right|= \begin{cases}1, & \sqrt{\omega_{1}^{2}+\omega_{2}^{2}} \leqslant 0.4 \pi \\ 0, & 0.6 \pi \leqslant \sqrt{\omega_{1}^{2}+\omega_{2}^{2}} \leqslant \pi\end{cases}
$$

where $T_{1}=T_{2}=1$.

By taking $L=M=21$ and assuming that the amplitude response varies linearly with the radius in the transition band, the sample amplitude response is given by a $21 \times 21$ matrix as

$$
A=\left[\begin{array}{llllllllll}
1 & 1 & 1 & 1 & 1 & 1 & 1 & 1 & 1 & .75 \\
1 & 1 & 1 & 1 & 1 & 1 & 1 & 1 & .75 & .5 \\
1 & 1 & 1 & 1 & 1 & 1 & 1 & 1 & .75 & .5 \\
1 & 1 & 1 & 1 & 1 & 1 & 1 & .75 & .5 & .25 \\
1 & 1 & 1 & 1 & 1 & 1 & 1 & .75 & .5 & .25 \\
1 & 1 & 1 & 1 & 1 & 1 & .75 & .5 & .25 & 0 \\
1 & 1 & 1 & 1 & 1 & .75 & .5 & .25 & 0 & 0 \\
1 & 1 & 1 & .75 & .75 & .5 & .25 & 0 & 0 & 0 \\
1 & .75 & .75 & .5 & .5 & .25 & 0 & 0 & 0 & 0 \\
.75 & .5 & .5 & .25 & .25 & 0 & 0 & 0 & 0 & 0 \\
.5 & .25 & .25 & 0 & 0 & 0 & 0 & 0 & 0 & 0 \\
.25 & 0 & 0 & 0 & 0 & 0 & 0 & 0 & 0 & 0 \\
0 & 0 & 0 & 0 & 0 & 0 & 0 & 0 & 0 & 0 \\
0 & 0 & 0 & 0 & 0 & 0 & 0 & 0 & 0 & 0 \\
0 & 0 & 0 & 0 & 0 & 0 & 0 & 0 & 0 & 0 \\
0 & 0 & 0 & 0 & 0 & 0 & 0 & 0 & 0 & 0 \\
0 & 0 & 0 & 0 & 0 & 0 & 0 & 0 & 0 & 0 \\
0 & 0 & 0 & 0 & 0 & 0 & 0 & 0 & 0 & 0 \\
0 & 0 & 0 & 0 & 0 & 0 & 0 & 0 & 0 & 0 \\
0 & 0 & 0 & 0 & 0 & 0 & 0 & 0 & 0 & 0 \\
0 & 0 & 0 & 0 & 0 & 0 & 0 & 0 & 0 & 0
\end{array}\right.
$$

The ideal amplitude response of the 2-D filter is shown in Fig. 4.

An easy-to-use, numerically reliable software package, called MATLAB, has been used to perform SVD on matrices $A, E_{1}$, and $E_{2}$ in order to obtain the necessary data for the design of the main section and the first two correction sections.

It is worth noting that while vector $\phi_{1}\left(=\gamma_{1}\right)$ obtained from the SVD of $A$ is a typical sampled amplitude specification for a 1-D low-pass filter, the data given by the SVD of error matrices $E_{1}$ and $E_{2}$ lead to the necessity of synthesizing 1-D filters with fairly arbitrary amplitude specifications. For example, given $A$ as in (22), the square root of $\phi_{1}$ is obtained as

$$
\begin{aligned}
& \phi_{1}^{1 / 2}=\left(\begin{array}{llllll}
1.0415 & 1.0263 & 1.0263 & 1.0005 & 0.9625 & 0.9120
\end{array}\right. \\
& \begin{array}{lllll}
0.8300 & 0.7075 & 0.5514 & 0.3705 & 0.1866
\end{array} \\
& \begin{array}{llllllllll}
0.0 & 0.0 & 0.0 & 0.0 & 0.0 & 0.0 & 0.0 & 0.0 & 0.0 & 0.0)^{\prime}
\end{array} \text {. }
\end{aligned}
$$

Now if a fourth-order approximation is obtained for trans- fer function $\bar{f}_{1}\left(z_{1}\right)$, the SVD of $E_{1}$ gives

$$
\begin{aligned}
& \tilde{\phi}_{2}^{1 / 2}=\left(\begin{array}{llllll}
1.0782 & 0.9881 & 0.9881 & 0.8452 & 0.8450 & 0.6482
\end{array}\right. \\
& \begin{array}{llllll}
0.3966 & 0.0270 & 0.1632 & 0.4371 & 0.6197
\end{array} \\
& \begin{array}{lllll}
0.7437 & 0.7916 & 0.7916 & 0.7916 & 0.7916
\end{array} \\
& \begin{array}{llllll}
0.7916 & 0.7916 & 0.7916 & 0.7916 & 0.7916)^{\prime} .
\end{array}
\end{aligned}
$$

Evidently, this represents an irregular amplitude specification. With this problem as a motivation, an optimization method has been developed which can be used for the design of 1-D digital filters satisfying arbitrary amplitude specifications.

By using the procedure in Section III along with the error compensation technique in Section IV and the aforementioned 1-D optimization method, a 2-D zero-phase filter comprising a main section and two correction sections has been designed for the amplitude specification in

$\left.\begin{array}{lllllllllll}.5 & .25 & 0 & 0 & 0 & 0 & 0 & 0 & 0 & 0 & 0 \\ .25 & 0 & 0 & 0 & 0 & 0 & 0 & 0 & 0 & 0 & 0 \\ .25 & 0 & 0 & 0 & 0 & 0 & 0 & 0 & 0 & 0 & 0 \\ 0 & 0 & 0 & 0 & 0 & 0 & 0 & 0 & 0 & 0 & 0 \\ 0 & 0 & 0 & 0 & 0 & 0 & 0 & 0 & 0 & 0 & 0 \\ 0 & 0 & 0 & 0 & 0 & 0 & 0 & 0 & 0 & 0 & 0 \\ 0 & 0 & 0 & 0 & 0 & 0 & 0 & 0 & 0 & 0 & 0 \\ 0 & 0 & 0 & 0 & 0 & 0 & 0 & 0 & 0 & 0 & 0 \\ 0 & 0 & 0 & 0 & 0 & 0 & 0 & 0 & 0 & 0 & 0 \\ 0 & 0 & 0 & 0 & 0 & 0 & 0 & 0 & 0 & 0 & 0 \\ 0 & 0 & 0 & 0 & 0 & 0 & 0 & 0 & 0 & 0 & 0 \\ 0 & 0 & 0 & 0 & 0 & 0 & 0 & 0 & 0 & 0 & 0 \\ 0 & 0 & 0 & 0 & 0 & 0 & 0 & 0 & 0 & 0 & 0 \\ 0 & 0 & 0 & 0 & 0 & 0 & 0 & 0 & 0 & 0 & 0 \\ 0 & 0 & 0 & 0 & 0 & 0 & 0 & 0 & 0 & 0 & 0 \\ 0 & 0 & 0 & 0 & 0 & 0 & 0 & 0 & 0 & 0 & 0 \\ 0 & 0 & 0 & 0 & 0 & 0 & 0 & 0 & 0 & 0 & 0 \\ 0 & 0 & 0 & 0 & 0 & 0 & 0 & 0 & 0 & 0 & 0 \\ 0 & 0 & 0 & 0 & 0 & 0 & 0 & 0 & 0 & 0 & 0 \\ 0 & 0 & 0 & 0 & 0 & 0 & 0 & 0 & 0 & 0 & 0 \\ 0 & 0 & 0 & 0 & 0 & 0 & 0 & 0 & 0 & 0 & 0\end{array}\right]$

(22). Fourth-order transfer-function approximations were used for the various subfilters. The realization of the 2-D filter is of the form depicted in Fig. 3, and the transfer functions of the subfilters are given in Table I. The amplitude responses of (a) the main section, (b) the main section plus the first correction section, and (c) the main section plus the two correction sections are depicted in Fig. 5(a)-(c).

The overall accuracy of the 2-D digital filter depends critically on the quality of the 1-D approximations obtained for the various subfilters. Consequently, if higher order approximations are used, better results are achieved. To illustrate this feature, the above 2-D filter was redesigned using sixth-order subfilters and the same number of correction sections. The transfer functions obtained for the subfilters are given in Table II. The amplitude responses of (a) the main section, (b) the main section plus the first correction section, and (c) the main section plus the two correction sections are depicted in Fig. 6(a)-(c). As can be 


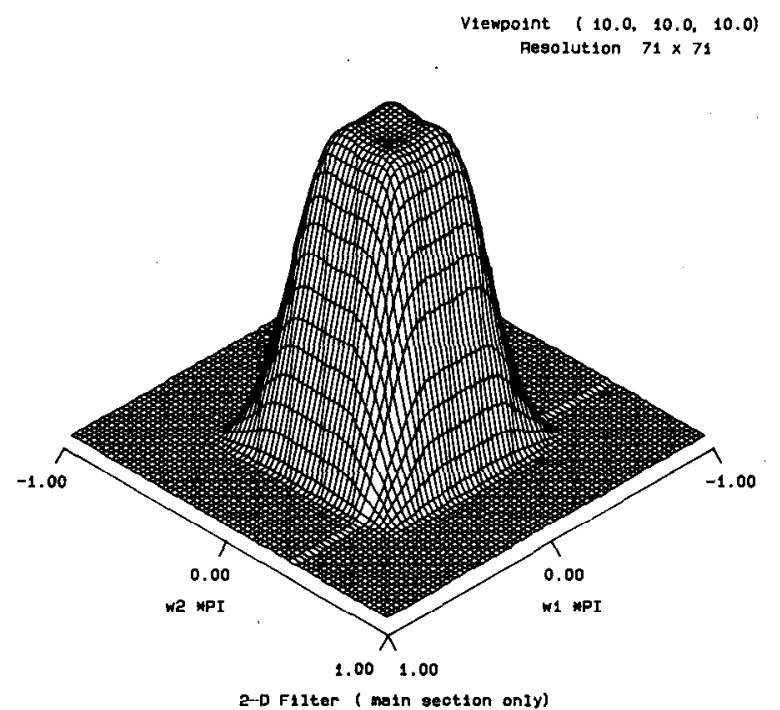

(a)

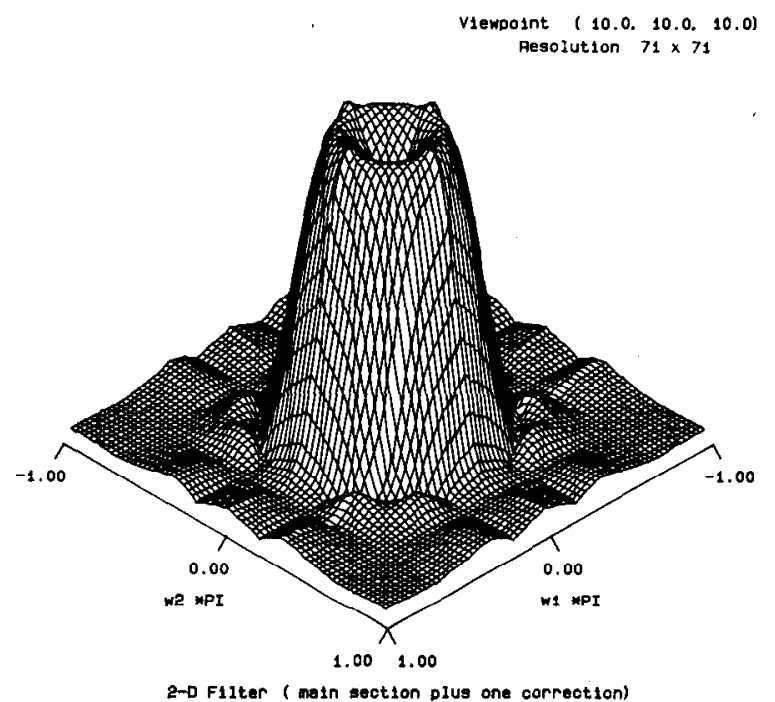

(b)

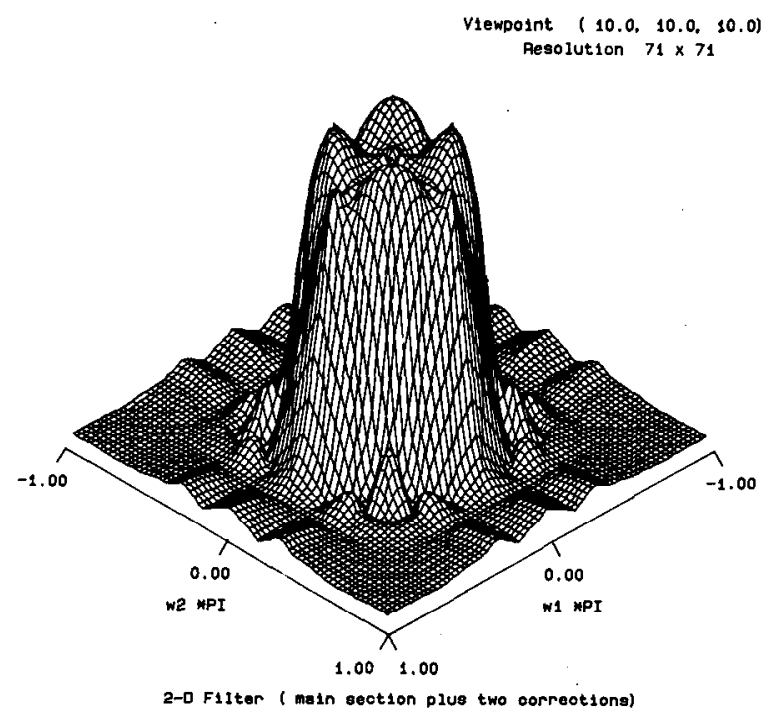

(c)

Fig. 5. Amplitude responses of 2-D digital filter using fourth-order subfilters. (a) Main section. (b) Main section plus one correction section. (c) Main section plus two correction sections.

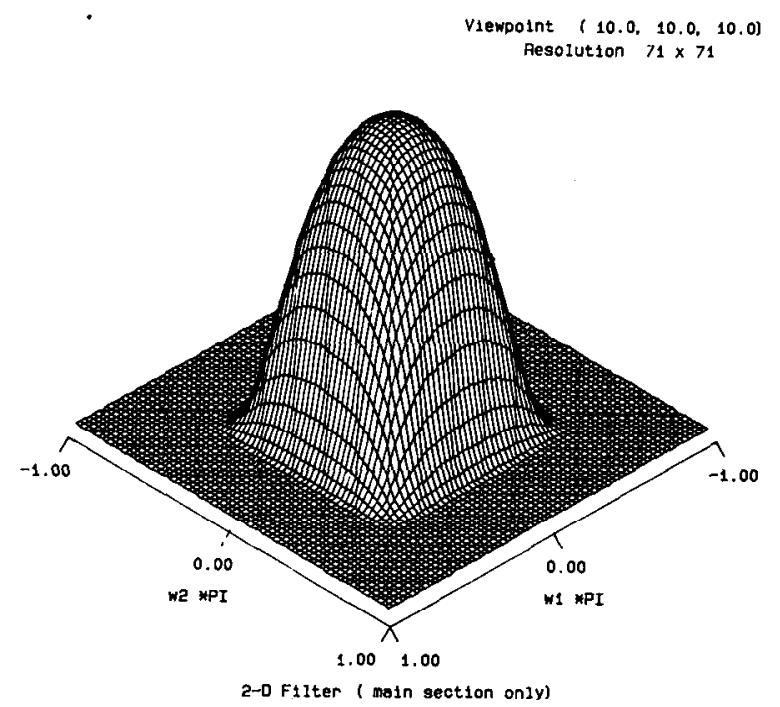

(a)

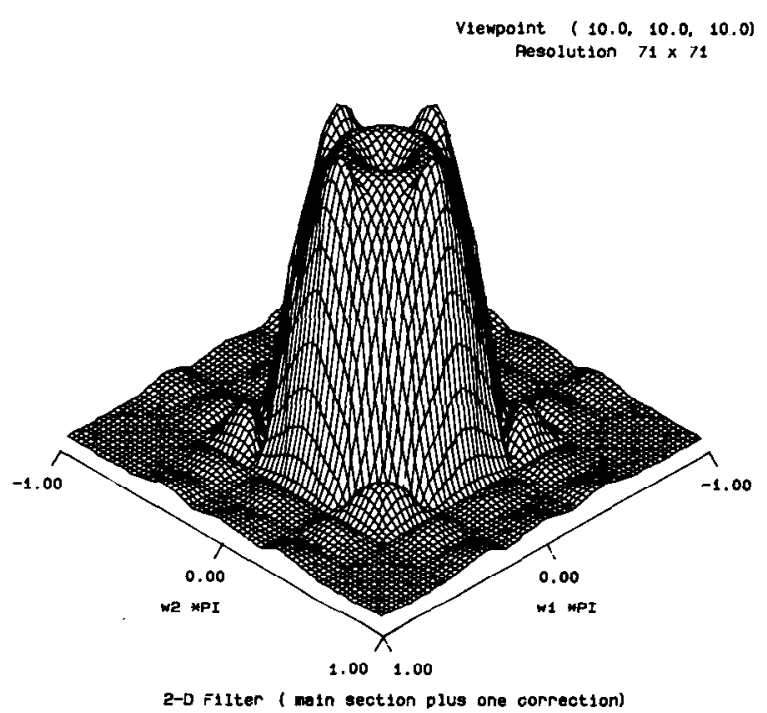

(b)

V1empoint $(10.0 .10 .0,10.0)$ Fesolution $71 \times 71$

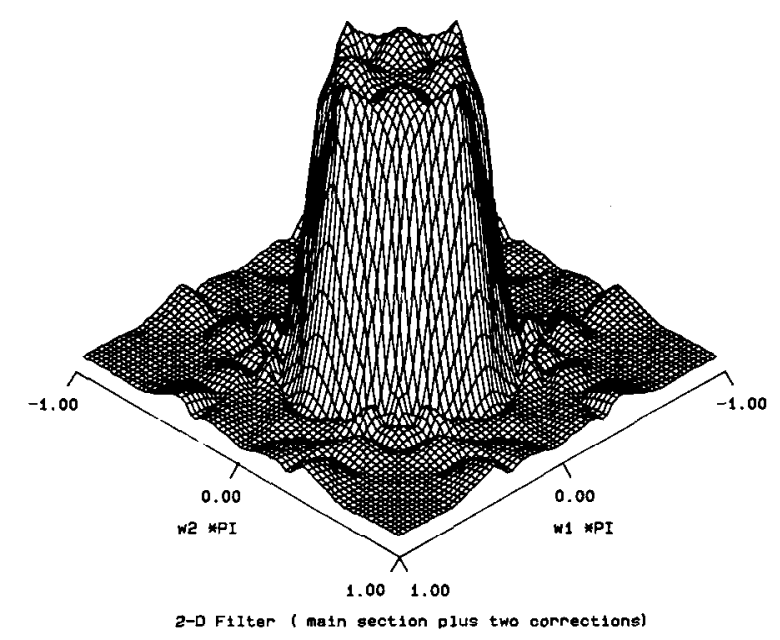

(c)

Fig. 6. Amplitude responses of 2-D digital filter using sixth-order subfilters. (a) Main section. (b) Main section plus one correction section. (c) Main section plus two correction sections. 
TABLE I

DEsign BASEd ON FoURTH-ORder SUBfilters

\begin{tabular}{|c|c|}
\hline The Main Section & $\bar{f}_{1}(z)=0.1255 \frac{\left(z^{2}+0.7239 x+1\right)\left(z^{2}+1.6343 z+1\right)}{\left(x^{2}+0.1367 z+0.6181\right)\left(z^{2}-0.5328 z+0.2278\right)}$ \\
\hline The First Correction Section & $\begin{aligned} \bar{f}_{2}(z) & =0.6098 \frac{\left(z^{2}+1.1618 z+0.1661\right)\left(z^{2}-0.8367 z+0.9958\right)}{\left(z^{2}+0.0963 z\right)\left(z^{2}-0.6124 z+0.32\right)} \\
\phi_{2}^{-} & =0.6266\end{aligned}$ \\
\hline The Second Correction Section & $\begin{aligned} \bar{f}_{3}(z) & =0.463 \frac{\left(x^{2}-1.5381 z-0.4456\right)\left(z^{2}-1.397 z+1.1191\right)}{\left(x^{2}-2.0408 z+1\right)\left(z^{2}-0.7092 z+0.6061\right)} \\
\phi_{3}^{-} & =0.2764\end{aligned}$ \\
\hline
\end{tabular}

TABLE II

Design Based on Sixth-Order Subfilters

\begin{tabular}{|c|c|}
\hline The Main Section & $\bar{f}_{1}(z)=0.07283 \frac{\left(x^{2}+0.6856 z+0.0946\right)\left(z^{2}+1.8510 z+0.0733\right)\left(z^{2}+1.0838 z+0.8708\right)}{\left(z^{2}+0.1603 x+0.7407\right)\left(z^{2}+0.2463 z-0.0669\right)\left(x^{2}-0.3816 z+0.3328\right)}$ \\
\hline The First Correction Section & $\begin{aligned} \bar{f}_{2}(z) & =0.008458 \frac{\left(z^{2}-0.8792 x+0.9938\right)\left(z^{2}+11.3798 z-35.0945\right)\left(z^{2}-1.1345 z+2.0328\right)}{\left(z^{2}-1.0015 z-0.6425\right)\left(z^{2}-0.5621 x-0.01960\right)\left(z^{2}-0.03034 x-0.4651\right)} \\
\phi_{2}^{-} & =0.6416\end{aligned}$ \\
\hline The Second Correction Section & $\begin{aligned} \bar{f}_{3}(z) & =0.01874 \frac{\left(z^{2}-1.3384 x+1.0258\right)\left(x^{2}-2.5942 x+12.6556\right)\left(z^{2}-0.1167 z+1.9796\right)}{\left(x^{2}-0.9051 x-0.7517\right)\left(z^{2}-0.3489 z-0.24\right)\left(z^{2}-0.05435 z+0.6863\right)} \\
\phi_{3}^{-} & =0.2985\end{aligned}$ \\
\hline
\end{tabular}

TABLE III

Filter Specifications Achieved

\begin{tabular}{|c|c|c|c|c|}
\hline & \multicolumn{2}{|c|}{$\begin{array}{c}\text { Filter Using 4-th Order } \\
\text { Subfilters }\end{array}$} & \multicolumn{2}{|c|}{$\begin{array}{c}\text { Filter Using 6-th Order } \\
\text { Subfilters }\end{array}$} \\
\hline Ripple in the passband & $1.5 \mathrm{~dB}$ & & $1.3 \mathrm{~dB}$ & \\
\hline $\begin{array}{l}\text { Minimum Stopband } \\
\text { Attenuation }\end{array}$ & $16 \mathrm{~dB}$ & & $22 \mathrm{~dB}$ & \\
\hline $\begin{array}{l}\text { Maximum Approximation } \\
\text { Error in the Passband }\end{array}$ & $\begin{array}{l}\text { Main Section: } \\
\text { With One Correction } \\
\text { Section: } \\
\text { With Two Correction } \\
\text { Sections: }\end{array}$ & $\begin{array}{l}0.4132 \\
0.1634 \\
0.0754\end{array}$ & $\begin{array}{l}\text { Main Section: } \\
\text { With One Correction } \\
\text { Section: } \\
\text { With Two Correction } \\
\text { Sections: }\end{array}$ & $\begin{array}{r}0.3789 \\
0.1149 \\
0.0724\end{array}$ \\
\hline $\begin{array}{l}\text { Maximum Approximation } \\
\text { Error in the Stopband }\end{array}$ & $\begin{array}{l}\text { Main Section: } \\
\text { With One Correction } \\
\text { Section: } \\
\text { With Two Correction } \\
\text { Sections: }\end{array}$ & $\begin{array}{l}0.3695 \\
0.1814 \\
0.1513\end{array}$ & $\begin{array}{l}\text { Main Section: } \\
\text { With One Correction } \\
\text { Section: } \\
\text { With Two Correction } \\
\text { Sections: }\end{array}$ & $\begin{array}{l}0.4134 \\
0.1564 \\
0.1088\end{array}$ \\
\hline
\end{tabular}


seen by comparing Figs. 5(c) and 6(c), the use of higher order approximations leads to a significant improvement in the overall amplitude response of the 2-D filter. The filter specifications achieved are summarized in Table III.

\section{Conclusions}

A method for the design of quadrantally symmetric 2-D digital filters has been proposed. Like the method of Twogood and Mitra, our method is based on the singular value decomposition, but through the use of parallel correction sections, prescribed amplitude responses can be obtained with a much higher degree of precision.

A degree of flexibility is inherent in the method in that the passband ripple can be reduced and the minimum stopband attenuation can be increased by increasing the number of correction sections and/or by increasing the orders of the various subfilters.

The design can be accomplished in terms of recursive zero-phase or nonrecursive linear-phase 1-D subfilters. In the first case, high-selectivity 2-D filters can be designed whose implementation entails minimal computational effort. In the second case, causal 2-D filters can be designed which can be used in real-time applications. In both cases, the processing delay is low since the filter is realized in terms of a number of parallel sections.

An important merit of the method is that the required 2-D digital filter is decomposed into a set of 1-D digital subfilters, which are much easier to design by optimization than the original 2-D digital filter.

\section{REFERENCES}

[1] J. L. Shanks, S. Treitel, and J. H. Justice, "Stability and synthesis of two-dimensional recursive filters," IEEE Trans. Audio Electroacoust., vol. AU-20, pp. 115-128, June 1972.

[2] J. M. Costa and A. N. Venetsanopoulos, "Design of circularly symmetric two-dimensional recursive filters," IEEE Trans. Acoustics, Speech, Signal Process., vol. ASSP-22, pp. 432-443, Dec. 1974

[3] N. A. Pendergrass, E. I. Jury, and S. K. Mitra, "Special transformations for two-dimensional digital filters," IFEE Trans. Circuits Syst., vol. CAS-23, pp. 26-35, Jan. 1976.

[4] M. Ahmadi, A. G. Constantinides, and R. A. King, "Design technique for a class of stable two-dimensional recursive digital filters," in Proc. 1976 IEEE Int. Conf. Acoustics, Speech, Signal Process., pp. 145-147.

[5] H. Chang and J. K. Aggarwal, "Design of two-dimensional recursive filters by interpolation," IEEE Trans. Circuits Syst, vol. CAS24, pp. 281-291, June 1977.

[6] D. M. Goodman, "A design technique for circularly symmetric low-pass filtcrs," IEEE Trans. Acoustics, Speech, Signal Process., vol. ASSP-26, no. 4, pp. 290-304, Aug. 1978.

[7] G. A. Maria and M. M. Fahmy, "An Lp design technique for two-dimensional digital recursive filters," IEEE Trans. Acoustics, Speech, Signal Process., vol. ASSP-22, pp. 15-21, Feb. 1974.

[8] C. Charalambous, "Design of 2-dimensional circularly symmetric digital filters," Proc. Inst. Elec. Eng., vol. 129, pt. G, no. 2, pp. 47-54, Apr. 1982.

[9] B. G. Mertzios and A. N. Venetsanopoulos, "Design of two-dimensional half-plane recursive digital filters with octagonal symmetry," Circuits Syst. Signal Process., vol. 4, no. 4, pp. 459-483, 1985.

[10] P. A. Ramamoorthy and L. T. Bruton, "Design of stable twodimensional analog and digital filters with applications in image processing," Int. J. Circuit Theory Appl., vol. 7, pp. 229-246, Apr. 1979.
[11] M. Ahmadi and V. Ramachandran, "A method for the design of stable $(N-D)$ analog and digital filters," in Proc. 1981 IEEE Int. Conf. Acoustics, Speech, Signal Process., pp. 704-707.

[12] S. Treitel and J. L. Shanks, "The design of multistage separable planar filters," IEEE Trans. Geosci. Electron, vol. GE-9, pp. 10-27, 1971.

[13] R. E. Twogood and S. K. Mitra, "Computer-aided design of separable two-dimensional digital filters," IEEE Trans. Acoustics, Speech, Signal Process., vol. ASSP-25, no. 2, pp. 165-169, 1977.

[14] P. K. Rajan and M. N. S. Swamy, "Quadrantal symmetry associated with two-dimensional digital transfer functions," IEEE Trans. Circuits Syst., vol. CAS-25, pp. 340-343, June 1983.

[15] G. W. Stewart, Introduction to Matrix Computations. New York: Academic Press, 1973.

[16] P. Lancaster and M. Tismenetsky, The Theory of Matrices, 2nd ed. New York: Academic Press, 1985

[17] A. N. Venetsanopoulos and B. G. Mertzios, "A decomposition theorem and its implications to the design and realization of two-dimensional filters," IEEE Trans. Acoustics, Speech, Signal Process., vol. ASSP-33, pp. 1562-1575, 1985

[18] J. H. McClellan, T. W. Parks, and L. R. Rabiner, "A computer program for designing optimum FIR linear phase digital filters," IEEE Trans. Audio electroacoust., vol. AU-21, pp. 506-526, Dec. 1973.

[19] A. Antoniou, "New improved method for the design of weightedChebyshev, nonrecursive, digital filters, IEEE Trans. Circuits Syst., vol. CAS-30, pp. $740-750$, Oct 1983 .

[201 C. Charalambous, "Minimax optimization of recursive digital filters using recent minimax results," IEEE Trans. Acoustics, Speech, Signal Process., vol. ASSP-23, pp. 333-345, Aug. 1975.

[21] C. Charalambous and A. Antoniou, "Equalisation of recursive digital filters," Proc. Inst. Elec. Eng., vol. 127, pt. G, pp. 219-225, Oct. 1980.

[22] A. Antoniou, Digital Filters: Analysis and Design. New York: McGraw-Hill, 1979.

Andreas Antoniou (M'69-SM'79-F'82), for a photograph and a biography, please see page 10 of the January 1987 issue of this Transactions.

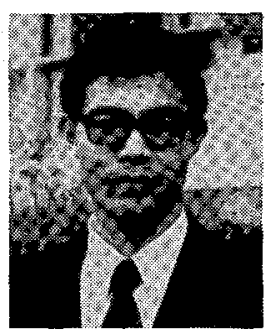

Wu-Sheng Lu (S'82-M'85) received the B.S. and M.S. degrees in mathematics from Fudan University and East China Normal University, China, in 1964 and 1980, respectively. He received the M.S. degree in electrical engineering and the $\mathrm{Ph}$.D. degree in control science from the University of Minnesota in 1983 and 1984, respectively.

He was a Postdoctoral Fellow at the University of Victoria, Canada, in 1985 and held a visiting position at the University of Minnesota in 1986. Since May 1987, he has been with the Department of Electrical and Computer Engineering, University of Victoria, where he is currently Associate Professor. His research interests include systems theory, analysis and synthesis of multidimensional digital filters, and robotics. 